\title{
An Approach to Temporal-Aware Procurement of Web Services *
}

\author{
Octavio Martín-Díaz, Antonio Ruiz-Cortés, \\ Amador Durán, and Carlos Müller \\ Dpto. Lenguajes y Sistemas Informáticos, \\ ETS. Ingeniería Informática - Universidad de Sevilla, \\ 41012 Sevilla (Spain - España) \\ \{octavio, aruiz\}@tdg.lsi.us.es \\ \{amador, cmuller\}@lsi.us.es
}

\begin{abstract}
In the context of web service procurement (WSP), temporalawareness refers to managing service demands and offers which are subject to validity periods, i.e. their evaluation depends not only on quality of service (QoS) values but also on time. For example, the QoS of some web services can be considered critical in working hours (9:00 to 17:00 from Monday to Friday) and irrelevant at any other moment. Until now, the expressiveness of such temporalaware specifications has been quite limited. As far as we know, most proposals have considered validity periods to be composed of a single temporal interval. Other proposals, which could allow more expressive time-dependent specifications, have not performed a detailed study about all the underlying complexities of such approach, in spite of the fact that dealing with complex expressions on temporality is not a trivial task at all. As a matter of fact, it requires a special design of the so-called procurement tasks (consistency and conformance checking, and optimal selection). In this paper, we present a constraint-based approach to temporal-aware WSP. Using constraints allows a great deal of expressiveness, so that not only demands and offers can be assigned validity periods but also their conditions can be assigned (possibly multiple) validity temporal subintervals. Apart from revising the semantics of procurement tasks, which we previously presented in the first edition of the ICSOC conferences, we also introduce the notion of the covering set of a demand, a topic which is closely related to temporality.
\end{abstract}

Keywords: services, procurement, quality, temporality, constraint programming.

\section{Introduction}

Web service procurement (WSP) — including automated search and selection-of the best web services according to their offered quality of service (QoS) is an activity which is gaining importance in the development of enterprise-level systems with a serviceoriented architecture (SOA) [18,24].

\footnotetext{
* This work has been funded by the Spanish Government under grant TIC2003-02737-C02-01, AGILWEB project.
} 
Web services, as a particular case of software packages, must be selected according to user requirements [3, 4]. On the one hand, these user requirements, to which we refer to as demands, are usually specified using boolean expressions, i.e. conditions on attributes describing the desired QoS of a service, for example 11 MTF $\geq 100$. On the other hand, web service providers usually guarantee the QoS of the service they provide, i.e. their offers, for example $100 \leq M T T F \leq 120$.

Procurement is the process of finding the best offer for a given demand [18]. Its typical scenario is: (1) a provider advertises its offers in a repository, (2) a customer asks its matchmaker for an offer to meet its demands, and (3) the matchmaker searches for matching offers, returning a result which may be an optimal offer according to a given customer criterion, or a failure message if no matching offer is found [21].

Temporality is an important aspect of WSP. If a demand or offer is subject to a validity period, it is said to be temporal-aware. As an example, in order to specify a (part of a) demand as "the MTTF of the web service at working hours (9:00 to 17:00, Monday to Friday) should be (at least) of 99\%, otherwise 90\%", we would require to define multiple, periodical validity periods associated to concrete conditions of the demand. Other temporal aspects to be taken into consideration are the granularity of time points, periods and durations, and the different time zones in which demands and offers (D\&O) can be available.

Not only it is necessary to extend the current models in WSP in order to improve their expressivess regarding temporality, but it is also needed to re-think the so-called procurement tasks, i.e. consistency and conformance checkings, and optimal selection, because of the non-trivial, intrinsic semantics of temporal expressions. For example, in a non-temporal-aware context we define the notion of pessimistic conformance so that an offer is conformant to a demand iff all the quality values guaranteed by the offer satisfy the conditions imposed by the demand. Let imagine a dummy demand and offer which were constituted by only a validity period, with no conditions regarding any quality attribute. If the validity period of the offer were included in the validity period of the demand, then such offer could be considered as conformant. But this is not the case, because the offer does not cover the validity period of the demand, so the offer is not conformant. In general, if temporality is taken into account, the notions of consistency, conformance, and optimal selection must be revised.

Until now - to the best of our knowledge - proposals allow a demand or an offer to have a validity period composed of only a single temporal interval. Only a few of them allows more complex temporal expressions, but most of them have not provided a detailed study about the underlying complexities of operations due to temporal semantics.

In this paper, we present an approach to temporal-aware WSP which is based on constraint programming $(\mathrm{CP})$. It is based on notions introduced in our previous nontemporal-aware, constraint-based approach to WSP [15, 18]. Using CP for WSP entails some advantages. First, D\&O can be stated declaratively, endowing the symmetric model with a very powerful expressiveness so that D\&O can be specified with the same expressiveness. Thus, offers are not limited to single parameter-value pairs. Moreover,

\footnotetext{
${ }^{1}$ MTTF stands for mean time to failure.
} 


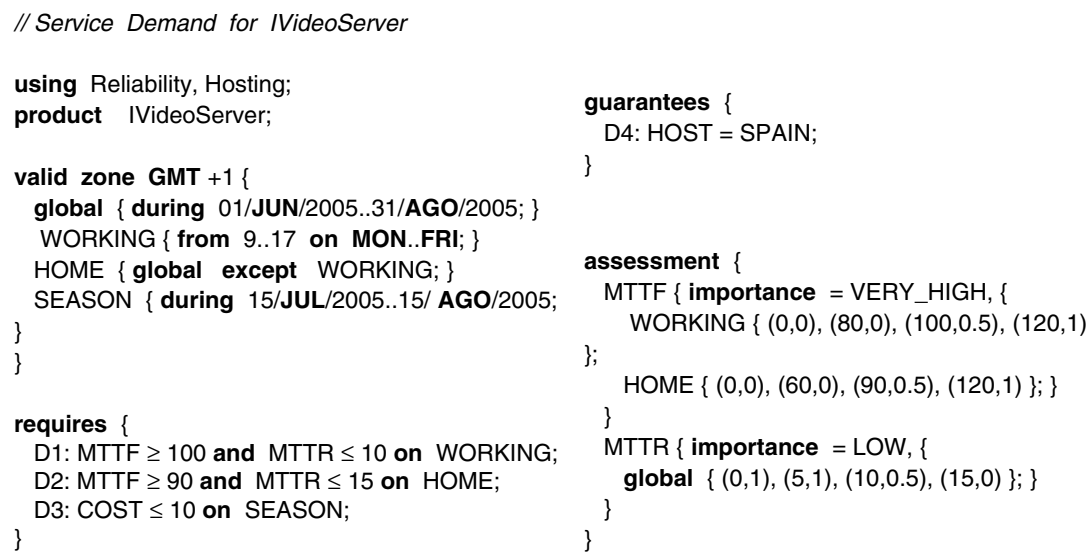

Fig. 1. An example of temporal-aware demand written in QRL

it is not necessary to write specific procedures for procurement tasks because they are implemented by checking properties of $\mathrm{D \& O}$ by means of a constraint solver.

Figure 1 shows an illustrative example of a temporal-aware demand. It is written in QRL (Quality Requirements Language), which is a language specifically devised for that purpose by one of the authors of this work as part of his $\mathrm{PhD}$ thesis [17]. This example is intended to be self-explanatory, in order to give an overview of the expressiveness of our approach. First, the demand establishes the Central Europe time zone (UTC/GMT+1). Then, it defines the global validity period (VP) together with other validity periods. The working hours VP is composed of some periodical temporal intervals, whereas the home hours VP is computed from the global VP and the previous one. Another valididy period is season which is non-periodical.

Note the validity periods can be assigned to conditions of the demand, so that the conditions on the same quality attributes are different at working hours or home hours. The season VP indicates the dates between which the cost of using the service should not be greater than $10 €$. The demand's host is always in Spain at any time of the global VP.

Note also the assessment criteria include utility functions which depends upon time. These functions are defined in a piecewise-like way. Each point is associated to the corresponding utility value (between 0 and 1 ), so that two consecutive points form a segment of the function. Utility functions are weighted by their grades of importance.

We also introduce the notion of covering. Since it is possible that none of the available offers were conformant to a given demand because they did not cover it, one could think of selecting several offers which are grouped together, in order to build a conformant offer which covers the validity period of the demand.

The rest of the paper is structured as follows. First, Section 2 introduces the theoretical basis for interpreting the temporal-aware procurement tasks by means of CSP, so that Section 3 presents our proposal to model them. Next, Section 4 provides a review of the state-of-the-art. Finaly, Section 5 concludes the paper and presents the future work. 


\section{Constraint Programming in a Nutshell}

Constraint programming (CP) is the study of computational models and systems based on contraints. $\mathrm{CP}$ is becoming a very interesting alternative to the modeling of optimization problems because of its potential to solve hard, real-life problems, and its declarative nature. A problem expressed as a set of constraints is formalized as a contraint satisfaction (optimization) problem (CSP) [5, 7, 8].

\subsection{Basic Definitions}

In this section, we introduce $\mathrm{CP}$ as the underlying formalism of our approach for expressing D\&O. The core of our proposal was a set of definitions used to rigorously define the so-called procurement tasks.

Definition 1 (CSP). A CSP is a three-tuple of the form $(V, D, C)$ where $V \neq \emptyset$ is a finite set of variables, $D \neq \emptyset$ is a finite set of domains (one for each variable) and $C$ is a set of constraints defined on $V$.

For instance, for the following $\operatorname{CSP}(\{x, y\},\{[0 . .2],[0 . .2]\},\{x+y<4, x-y \geq 1\})$, the assignment $\sigma=\{x \mapsto 2, y \mapsto 0\}$ is one of its solutions.

Definition 2 (Solution Space). Let $\psi$ be a CSP of the form $(V, D, C)$, its solution space, denoted as $\operatorname{sol}(\psi)$, is composed of all its possible solutions.

$$
\operatorname{sol}(\psi)=\{\sigma \in V \rightarrow D \mid \sigma(C)\}
$$

where $\sigma(C)$ holds iff each assignment in $\sigma$ satisfies every constraint in $C$.

In the previous example the solution space is $\{\{x \mapsto 1, y \mapsto 0\},\{x \mapsto 2, y \mapsto 0\}$, $\{x \mapsto 2, y \mapsto 1\}\}$.

Definition 3 (Satisfiability). Let $\psi$ be a CSP of the form $(V, D, C), \psi$ is said to be satisfiable, denoted as sat $(\psi)$, iff its solution space is not empty.

$$
\operatorname{sat}(\psi) \Leftrightarrow \operatorname{sol}(\psi) \neq \emptyset
$$

Definition 4 (Minimum Space and Value). Let $\psi$ be a CSP of the form $(V, D, C)$, its minimum space with regard to an objective function $O$, denoted as $\min _{S}(\psi, O)$, is composed of all the solutions of $\psi$ that minimize $O$. Its minimum value with regard to $O$, denoted as $\min _{V}(\psi, O)$, is the value the objective function takes on $\min _{S}(\psi, O)$.

$$
\begin{gathered}
\min _{S}(\psi, O)=\left\{\sigma \in \operatorname{sol}(\psi) \mid \forall \sigma^{\prime} \in \operatorname{sol}(\psi) \cdot O(\sigma) \leq O\left(\sigma^{\prime}\right)\right\} \\
\min _{V}(\psi, O)=m \Leftrightarrow \forall \sigma \in \min _{S}(\psi, O) \cdot O(\sigma)=m
\end{gathered}
$$

For instance, consider the CSP in the previous example and an objective function defined as $O(x, y)=x^{2} y$. In this case, $\min _{S}(\psi, O)=\{\{x \mapsto 1, y \mapsto 0\}$, $\{x \mapsto 2, y \mapsto 0\}\}$. The minimum value is 0 . 


\subsection{Filters and Projections}

In general, the solution space of a CSP can be restricted by means of intersecting a second CSP.

Filters. A filter is a kind of selection, which allows to obtain a CSP whose solution space has been restricted to those solutions containing a (possibly partial) assignment over the variables.

Definition 5 (Filtering). Let $\psi$ be a CSP of the form $(V, D, C)$, and $\sigma_{\pi}=\left\{v_{1} \mapsto d_{1}, \ldots, v_{k} \mapsto d_{k}\right\}$ an assignment defined over the $k$ variables in $\pi \subseteq V$, the filtering of $\psi$ on $\sigma_{\pi}$, denoted as $\psi_{v_{1} \mapsto d_{1}, \ldots, v_{k} \mapsto d_{k}}$, is another CSP defined on $V$ and $D$ whose constraint set $C^{\prime}$ is $C$ wherein as many equality constraints as assignments in $\sigma_{\pi}$ have been added.

$$
C^{\prime}=C \cup \bigcup_{i=1}^{k}\left\{v_{i}=d_{i}\right\}
$$

In the previous example, the filtering over $\sigma_{\pi}=\{y \mapsto 0\}$ results in a CSP whose solution space is $\{\{x \mapsto 1, y \mapsto 0\},\{x \mapsto 2, y \mapsto 0\}\}$.

Projections. A projection is another kind of selection, which allows to obtain those values which take a set of variables whenever the CSP is satisfiable.

Definition 6 (Projection). Let $\psi$ be a CSP of the form $(V, D, C)$, and $\pi$ a set of variables such that $\pi \subseteq V$, the projection of $\psi$ over $\pi$, denoted as $\psi_{\Downarrow \pi}$, is another CSP defined on $\pi$ and $D_{\pi}$ whose solution space is composed of values of variables in $\pi$ which are part of any solution in $\operatorname{sol}(\psi)$.

$$
\operatorname{sol}\left(\psi_{\Downarrow \pi}\right)=\left\{\sigma_{\pi} \in \pi \rightarrow D_{\pi} \mid \exists \sigma \in \operatorname{sol}(\psi) \cdot \sigma_{\pi} \subseteq \sigma\right\}
$$

where $D_{\pi} \subseteq D$ is the set of domains of variables in $\pi$.

In the previous example, the projection of the solution space over $\pi=\{x\}$ results in $\{\{x \mapsto 1\},\{x \mapsto 2\}\}$.

\section{Temporal-Aware Procurement Using Constraint Programming}

In [15, 18], we described how CP can help automating the procurement tasks, i.e. the checking for consistency and conformance, and selection of optimal offers. The key to automating the procurement tasks is to map D\&O onto CSPs. In order to do so, each attribute must be mapped onto a variable with its corresponding domain, and each condition must be mapped onto a constraint.

In this section, we review these notions in order to make them temporal-aware. We assume a linear, discrete time-structure based on natural numbers. Time elements are point times and temporal intervals. A temporal interval is given by two time points representing their extremes. 


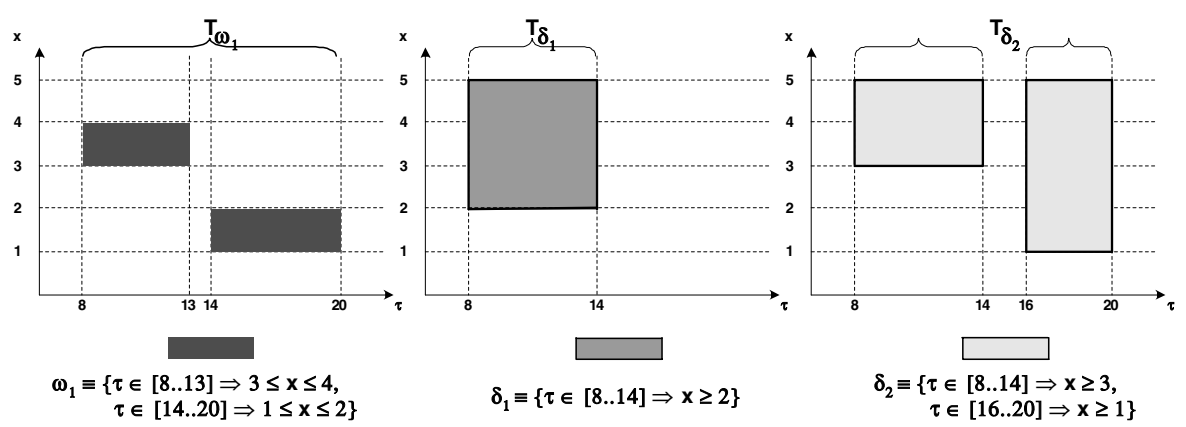

Fig. 2. Solution spaces of temporal-aware offers and demands

\subsection{Demands and Offers}

Demands assert the conditions the provider shall meet, whereas offers assert the conditions a provider guarantee 2 . Regarding temporality, all $\mathrm{D} \& \mathrm{O}$ are considered as (by default) temporal-aware, i.e. they all have a validity period and their inner conditions can (optionally) establish time-dependent demand requirements or offer guarantees. If a $\mathrm{D} \& \mathrm{O}$ does not have an explicit validity period, it will be supposed to have an infinite temporal interval.

Let $\delta$ denote a demand, and $\omega$ denote an offer. Their corresponding CSP are denoted as $\psi_{\delta}$ and $\psi_{\omega}$, respectively. Let $\alpha$ denote a demand or offer. Any demand or offer $\alpha$ has an (implicit) temporal variable, denoted as $\tau$, so that its domain $D_{\tau}$ corresponds to the validity period. Inner conditions of D\&O are based on QoS attributes and (eventually) the temporal variable, so that distinct temporal subintervals can be assigned to them, provided these subintervals are included in the validity period.

$T_{\alpha}$ stands for a CSP of the form $\left(\tau, D_{\tau}\right.$, true $)$ whose its solution space corresponds to the validity period of $\alpha$. Note $\tau^{\prime} \in T_{\alpha}$ is a shorthand for an assignment at time $\tau^{\prime}$ which belongs to the validity period.

For instance, the following tuples denote an offer $\omega_{1}$ and two demands $\delta_{1}$ and $\delta_{2}$ :

$$
\begin{gathered}
\omega_{1}=(\{x, \tau\},\{[0 . .5],[8 . .20]\},\{\tau \in[8 . .13] \Rightarrow 3 \leq x \leq 4, \tau \in[14 . .20] \Rightarrow 1 \leq x \leq 2\}) \\
\delta_{1}=(\{x, \tau\},\{[0 . .5],[8 . .14]\},\{\tau \in[8 . .14] \Rightarrow x \geq 2\}) \\
\delta_{2}=(\{x, \tau\},\{[0 . .5],[8 . .14] \cup[16 . .20]\},\{\tau \in[8 . .14] \Rightarrow x \geq 3, \tau \in[16 . .20] \Rightarrow x \geq 1\})
\end{gathered}
$$

Their solution spaces are shown graphically in Figure 2. The offer $\omega_{1}$ has the temporal interval $[8 . .20]$ as validity period, representing the office hours of a day. Each guarantee of this offer is assigned a temporal subinterval which is included in the validity period, covering the overrall temporal interval. The first guarantee of $\omega_{1}$ is valid at times in [8..13]. The second guarantee of $\omega_{1}$ is valid at times in [14..20].

Note the " $\Rightarrow$ " operator is the logic implication with its usual meaning.

\footnotetext{
${ }^{2}$ For the sake of simplicity, we are assuming a one-way matchmaking, i.e. demands only require something from offers, and offers only guarantee something to demands, but not viceversa. The interested reader is referred to [18] wherein a two-way matchmaking is presented.
} 


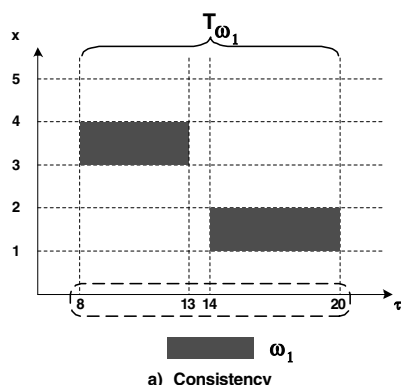

a) Consistency

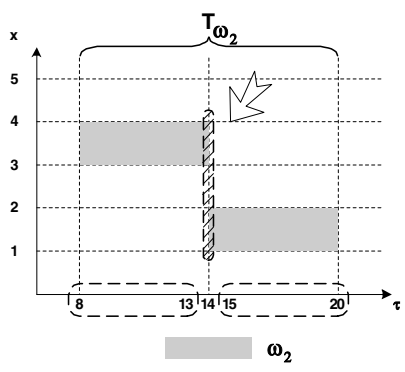

b) Non-consistency

Fig. 3. Temporal-aware consistency

The first demand $\delta_{1}$ has a unique requirement whose temporal subinterval is regarded to the overrall validity period [8..14]. This demand is not defined at any other time of a day.

The second demand $\delta_{2}$ has a validity period composed of two subintervals [8..14] and $[16 . .20]$ so that each requirement is assigned to every subinterval.

\subsection{Consistency}

Checking a demand or offer for consistency allows to unveil whether they have internal contradictions or not along the times whenever it is defined. If temporality is taken into account, consistency must also involve a checking of their validity periods. Moreover, since their requirements or guarantees can be also assigned one or more temporal intervals, they should be included in the validity period in order to be considered as consistent.

Note that it is also possible different demand requirements or offer guarantees have to be fulfilled at the same time. Checking the consistency of conditions and validity periods separately is not enough, but once validity periods have been checked, the consistency of conjunction of all demands requirements or offer guarantees at any time of the validity period has to be checked, as well.

Definition 7 (Consistency). A demand or offer $\alpha$ is said to be consistent iff the projection over time of its corresponding CSP $\psi_{\alpha}$ equals its non-empty validity period.

$$
\operatorname{consistent}(\alpha) \Leftrightarrow \operatorname{sol}\left(\psi_{\alpha \Downarrow \tau}\right)=\operatorname{sol}\left(T_{\alpha}\right)
$$

For instance, consider the offer $\omega_{1}$ in the previous example, and another offer $\omega_{2}$ defined on the same attributes and domains but with the following conditions:

$$
\{\tau \in[8 . .14] \Rightarrow 3 \leq x \leq 5, \tau \in[14 . .20] \Rightarrow 1 \leq x \leq 3\}
$$

Both of them are shown in Figure 3 Note that the offer $\omega_{1}$ is consistent (see Figure 3 a) because there are no contradictory conditions at any time in the validity period. However, the offer $\omega_{2}$ is not consistent (see Figure 3.b) because at time $\tau=14$ (marked with an arrow) there exist two contradictory conditions, so that the solution space of 
their conjuction at such a time is empty, and that point time is not included in the projection. Therefore, since the projection does not equal the validity period, the offer $\omega_{2}$ is not consistent.

\subsection{Conformance}

Checking if an offer conforms to a demand allows to know whether the values guaranteed by a party (the offer from a provider) meet the values required by the other party (the demand of a client) whenever the demand is defined. A non-temporal-aware offer $\omega$ and a non-temporal-aware demand $\delta$ is said to be pessimistic-conformant iff the solution space of $\psi_{\omega}$ is a subset of the solution space of $\psi_{\delta}$. In terms of CP, this can be expressed by means of Marriott and Stuckey expression [14]:

$$
\operatorname{conformant}(\omega, \delta) \Leftrightarrow \neg \operatorname{sat}\left(\psi_{\omega} \wedge \neg \psi_{\delta}\right)
$$

If temporality is taken into account, this checking must be carried out at any time of the validity period of the demand. In Section 1 , we have introduced the need of revising the conformance notion, so that if an offer and a demand were defined exclusively by their validity periods, then they would be considered as conformant iff the validity period of the offer covered the validity period of the demand.

Definition 8 (Conformance). An offer $\omega$ and a demand $\delta$ are said to be conformant iff the validity period of $\omega$ covers the validity period of $\delta$, and the projection over time of the CSP representing those solutions of $\omega$ which are not a solution of $\delta$ is disjoint to the validity period of $\delta$.

$$
\begin{aligned}
\operatorname{conformant}(\omega, \delta) \Leftrightarrow & \operatorname{sol}\left(T_{\delta}\right) \subseteq \operatorname{sol}\left(T_{\omega}\right) \\
& \wedge \operatorname{sol}\left(\left\{\psi_{\omega} \wedge \neg \psi_{\delta}\right\}_{\Downarrow \tau}\right) \cap \operatorname{sol}\left(T_{\delta}\right)=\emptyset
\end{aligned}
$$

For instance, consider the offer $\omega_{1}$ and the demand $\delta_{1}$ in the previous example, together with the demands $\delta_{3}$ and $\delta_{4}$ whose definitions are:

$$
\begin{aligned}
& \delta_{3}=(\{x, \tau\},\{[0 . .5],[8 . .20]\},\{\tau \in[8 . .13] \Rightarrow x \geq 3, \tau \in[14 . .20] \Rightarrow x \geq 1\}) \\
& \delta_{4}=(\{x, \tau\},\{[0 . .5],[7 . .20]\},\{\tau \in[7 . .13] \Rightarrow x \geq 3, \tau \in[14 . .20] \Rightarrow x \geq 1\})
\end{aligned}
$$

Their conformance relationships are shown in Figure 4 Note that the offer $\omega_{1}$ is not conformant to the demand $\delta_{1}$ (see Figure 4 a) because at $\tau=14$ (marked with an arrow) the solution space of the offer is not a subset of solution space of the demand. Note this situation is detected by the above formula, because the time $\tau=14$ belongs to the projection over time of those solutions of $\omega_{1}$ which are not included in the solution space of the demand $\delta_{1}$, and it is also included in its validity period $T_{\delta_{1}}$. The offer $\omega_{1}$ is conformant to the demand $\delta_{3}$ (see Figure 4, b) because it is conformant at any time of its validity period, covering it completely as well. Finally, the offer $\omega_{1}$ is not conformant to the demand $\delta_{4}$ (see Figure $4 \mathrm{c}$ ) because it does not cover its validity period since it does not supply anything at $\tau=7$ (marked with an arrow). The striped zones in Figure 4 represent the solution spaces of the negated CSP corresponding to the demands. 


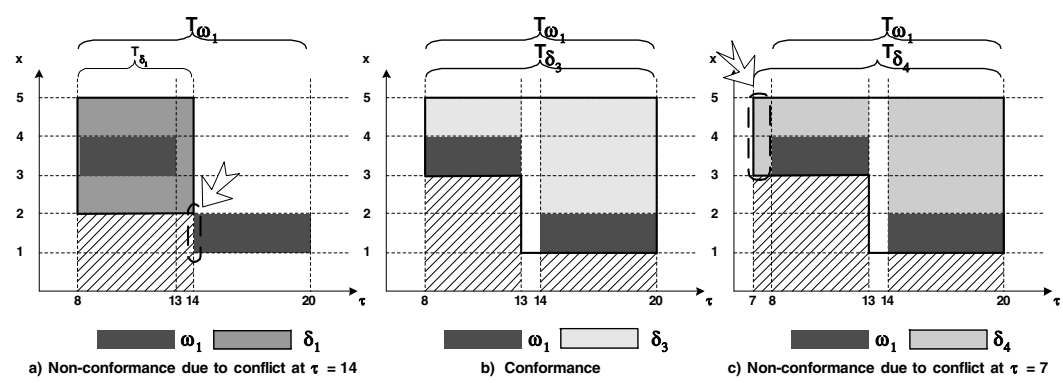

Fig. 4. Temporal-aware conformance

\subsection{Finding the Optimal Offers}

The final goal of matchmaking is, given a demand, finding a conformant offer that is optimal from the customer's point of view. This task is interpreted as a contraint satisfaction optimization problem (CSOP), which requires a preference order defined on the offer set. It is usual to establish such an order by means of a weighted composition of utility functions, whose general form is as follows:

$$
\mathcal{U}\left(a_{1}, \ldots, a_{n}\right)=\sum_{i=1}^{n} k_{i} U_{i}\left(a_{i}\right) \quad k_{i} \in[0,1] \quad \sum_{i=1}^{n} k_{i}=1
$$

where each $a_{i}$ denotes a quality attribute, each $k_{i}$ its associated weight, and each $U_{i}$ its associated utility function ranging over $[0,1]$ and describing how important the values of attribute are for the client.

Definition 9 (Set of Optimal Offers). Let $\Omega_{\delta}$ be a set of conformant offers to the demand $\delta$, and $\mathcal{U}$ the assessment criteria given by an utility function, the set of optimal offers, denoted as $\Omega_{\delta, \mathcal{U}}^{*}$, is constituted of those offers in $\Omega_{\delta}$ which maximize $\mathcal{U}$.

$$
\Omega_{\delta, \mathcal{U}}^{*}=\left\{\omega \in \Omega_{\delta} \mid \forall \omega^{\prime} \in \Omega_{\delta} \cdot \mathcal{U}(\omega) \geq \mathcal{U}\left(\omega^{\prime}\right)\right\}
$$

where $\mathcal{U}(\omega)$ stands for the utility of the offer $\omega$ given $\mathcal{U}$.

In a non-temporal-aware context, the utility of an offer corresponds to the worst case, that is to say, the utility of those values which minimize the utility function:

$$
\mathcal{U}(\omega)=\min _{V}\left(\psi_{\omega}, \mathcal{U}\right)
$$

If temporality is taken into account, utility functions can be dependent upon time, so that quality attributes can have different utility values at distinct temporal intervals. The utility of an offer is the average utility during the validity period of $\delta$ :

$$
\mathcal{U}(\omega)=\frac{1}{\left|\operatorname{sol}\left(T_{\delta}\right)\right|} \sum_{\tau^{\prime} \in T_{\delta}} \min _{V}\left(\psi_{\omega, \tau \mapsto \tau^{\prime}}, \mathcal{U}\right)
$$

where $\psi_{\omega, \tau \mapsto \tau^{\prime}}$ stands for the CSP which corresponds to $\omega$ filtered at time $\tau=\tau^{\prime}$. 

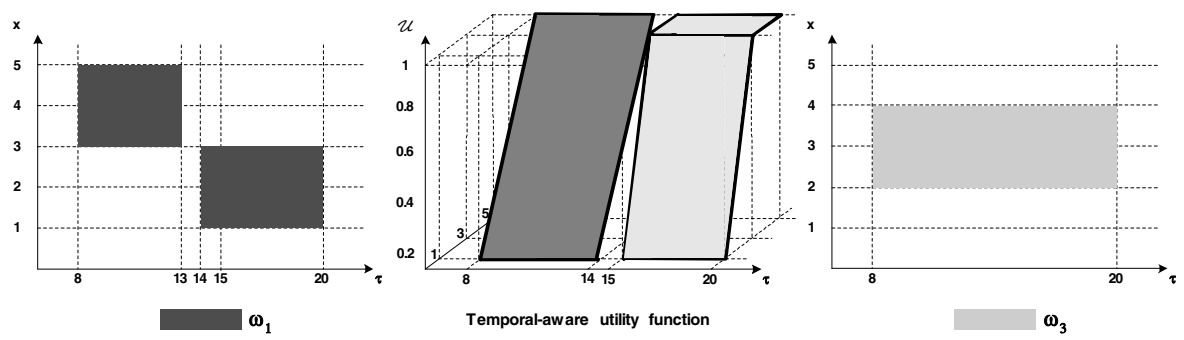

Fig. 5. Optimal selection with temporal-aware utility functions and offers

For instance, consider the offer $\omega_{1}$ in the previous example, and another offer $\omega_{3}$ defined on the same attributes and domains but with the following condition $\{\tau \in[8 . .20] \Rightarrow 2 \leq x \leq 3\}$. Assume these offers are conformant to a demand $\delta$ whose validity period is $[8 . .20]$, so that the assessment criteria is given by the utility function $\mathcal{U}$ in Figure 5 Note it gives different utility values for intervals $\tau \in[8 . .14]$ and $\tau \in[15 . .20]$. The set of optimal offers is $\Omega_{\delta, \mathcal{U}}^{*}=\left\{\omega_{3}\right\}$, according to their utility values:

$$
\begin{gathered}
\mathcal{U}\left(\omega_{1}\right)=\frac{1}{13}\left\{\frac{3}{5} \times 6+\frac{1}{5} \times 1+\frac{1}{3} \times 6\right\}=0.45 \\
\mathcal{U}\left(\omega_{3}\right)=\frac{1}{13}\left\{\frac{2}{5} \times 7+\frac{2}{3} \times 6\right\}=0.52
\end{gathered}
$$

The utility of $\omega_{1}$ is computed in this way. Note that the number of time points which belongs to $T_{\delta}$ is 13 . If $\tau \in$ [8..13] (six time points) then $x=3$ is given an utility of $3 / 5$, if $\tau=14$ (one time point) then $x=1$ is given an utility of $1 / 5$, and if $\tau \in[15 . .20]$ (another six time points) then $x=1$ is given an utility of $1 / 3$. The utility of $\omega_{3}$ is computed in a similar way.

\subsection{Finding the Optimal Covering}

Since it is possible that none of the available offers were conformant to a given demand because they did not cover it, one could think of selecting several offers so that all together are conformant to the demand, covering all the validity period. The covering problem is to find such a set of offers, optimizing according to assessment criteria from demand and other (optional) criteria, in order to adopt different strategies such as, for example, to minimize the number of offers.

Definition 10 (Covering). Let $\delta$ be a demand and $\Omega$ a set of available offers. $\Omega$ is said to be a covering set of $\delta$ iff there exists (at least) a conformant offer in $\Omega$ at any time of the validity period of the demand.

$$
i s \text { CoveringSet }(\Omega, \delta) \Leftrightarrow \forall \tau^{\prime} \in T_{\delta}, \exists \omega \in \Omega \cdot \operatorname{conformant}\left(\omega_{\tau \mapsto \tau^{\prime}}, \delta_{\tau \mapsto \tau^{\prime}}\right)
$$

where $\omega_{\tau \mapsto \tau^{\prime}}$ and $\delta_{\tau \mapsto \tau^{\prime}}$ stand for the offer $\omega$ and the demand $\delta$ at time $\tau=\tau^{\prime}$, respectively.

\footnotetext{
${ }^{3}$ An offer is available iff it provides the functionality required by a demand.
} 


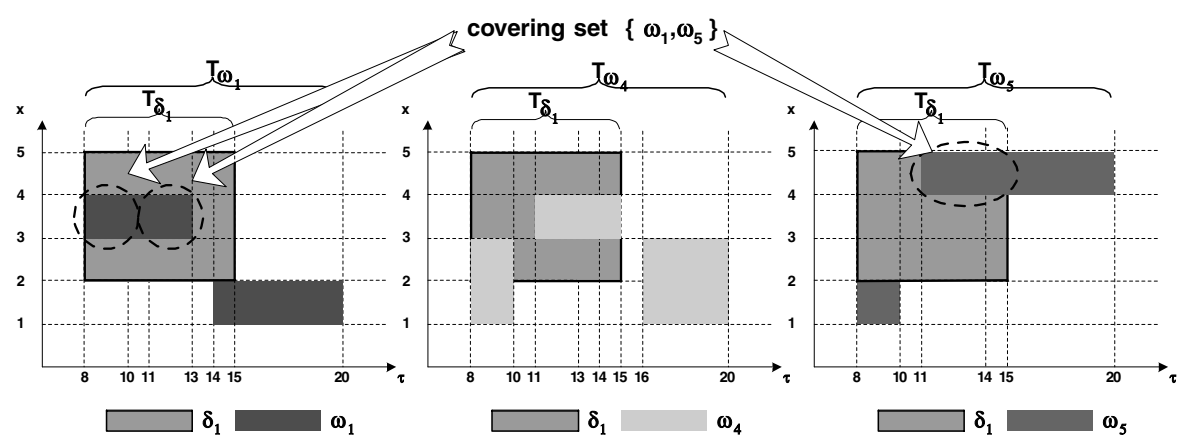

Fig. 6. Covering and temporal-awareness

For instance, consider the demand $\delta_{1}$ whose valid period is $T_{\delta_{1}}=[8 . .15]$ and the assessment criteria given by the utility function $\mathcal{U}$, and the offer $\omega_{1}$ in the previous example, together with the following offers $\omega_{4} \mathrm{y} \omega_{5}$ which are also defined on the same attributes and domains as $\omega_{1}$, but with the following constraints:

$$
\begin{gathered}
C_{\omega_{4}}=\{\tau \in[8 . .10] \Rightarrow 1 \leq x \leq 3, \tau \in[11 . .15] \Rightarrow 3 \leq x \leq 4, \tau \in[16 . .20] \Rightarrow 1 \leq x \leq 3\} \\
C_{\omega_{5}}=\{\tau \in[8 . .10] \Rightarrow 1 \leq x \leq 2, \tau \in[11 . .20] \Rightarrow 4 \leq x \leq 5\}
\end{gathered}
$$

The demand and offers are shown in Figure 6. None of them is conformant to the given demand, but if it were possible to join two (or more) of them, then one would be able to build a conformant offer, i.e. the so-called covering set of a demand.

Figure 6 shows the covering set of $\delta_{1}$ which is constituted by the offers $\left\{\omega_{1}, \omega_{5}\right\}$ (marked with the arrows). It is a covering set because at any time of $T_{\delta_{1}}$ there exist (at least) a conformant offer:

$$
\tau \in[8 . .10] \rightarrow \omega_{1} ; \tau \in[11 . .13] \rightarrow \omega_{1}, \omega_{5} ; \tau \in[14 . .15] \rightarrow \omega_{5}
$$

The sets of offers $\left\{\omega_{1}, \omega_{4}\right\}$ and $\left\{\omega_{1}, \omega_{4}, \omega_{5}\right\}$ also conform a covering set of the demand. However, the set of offers $\left\{\omega_{4}, \omega_{5}\right\}$ is not a covering set, because at time $\tau \in[8 . .10]$ there is no offer conformant to the demand.

Among all the covering sets which can be conformed from a set of offers, one should be able to select the best one. Therefore, we need to compute their utility according to assessment criteria attached to a demand.

Let $\Omega$ be a covering set of the demand $\delta_{1}$, and $\mathcal{U}$ the utility function of $\delta_{1}$, the utility of a covering set is given by the aggregation of maximum utilities at any time in the validity period of $\delta_{1}$ :

$$
\mathcal{U}(\Omega)=\frac{1}{\left|\operatorname{sol}\left(T_{\delta}\right)\right|} \sum_{\tau^{\prime} \in T_{\delta}} \max _{\omega \in \Omega_{\delta, \tau \mapsto \tau^{\prime}}}\left\{\min _{V}\left(\psi_{\omega, \tau \mapsto \tau^{\prime}}, \mathcal{U}\right)\right\}
$$

where $\Omega_{\delta, \tau \mapsto \tau^{\prime}}$ is the subset of offers in $\Omega$ which are conformant to $\delta$ at time $\tau=\tau^{\prime}$ :

$$
\Omega_{\delta, \tau \mapsto \tau^{\prime}}=\left\{w \in \Omega \mid \operatorname{conformant}\left(\omega_{\tau \mapsto \tau^{\prime}}, \delta_{\tau \mapsto \tau^{\prime}}\right)\right\}
$$


For instance, consider the set of offers $\Omega=\left\{\omega_{1}, \omega_{4}, \omega_{5}\right\}$ available to the demand $\delta_{1}$, whose assessment criteria is $\mathcal{U}$, in the previous example, the utility values of the covering sets of $\delta_{1}$ are:

$$
\begin{gathered}
\mathcal{U}\left(\left\{\omega_{1}, \omega_{4}\right\}\right)=\frac{1}{7}\left\{\frac{3}{5} \times 3+\frac{3}{5} \times 3+\frac{3}{5} \times 1+\frac{3}{3} \times 1\right\}=0.74 \\
\mathcal{U}\left(\left\{\omega_{1}, \omega_{5}\right\}\right)=\frac{1}{7}\left\{\frac{3}{5} \times 3+\frac{4}{5} \times 3+\frac{4}{5} \times 1+1 \times 1\right\}=0.86 \\
\mathcal{U}\left(\left\{\omega_{1}, \omega_{4}, \omega_{5}\right\}\right)=\frac{1}{7}\left\{\frac{3}{5} \times 3+\frac{4}{5} \times 3+\frac{4}{5} \times 1+1 \times 1\right\}=0.86
\end{gathered}
$$

The utility of the covering $\left\{\omega_{1}, \omega_{5}\right\}$ is computed in this way. Note that the number of time points which belongs to $T_{\delta}$ is 7 . If $\tau \in[8 . .10]$ (three time points) then the offer $\omega_{1}$ has the conformant value $x=3$ which is given an utility of $3 / 5$, if $\tau \in[11 . .13]$ (three time points) then both offers are conformant, but the best one is $\omega_{5}$ because it offers a conformant value $x=4$ which is given an utility of $4 / 5$ whereas $\omega_{1}$ has a conformant value $x=3$ which is given a worse utility of $3 / 5$, if $\tau=14$ (one time point) then the offer $\omega_{5}$ has the conformant value $x=4$ which is given an utility of $4 / 5$, and if $\tau=15$ (another one time point) then the offer $\omega_{5}$ has the conformant value $x=4$ which is given an utility of 1 . The utility of the remaining coverings is computed in a similar way.

Definition 11 (Set of Optimal Coverings). Let $\delta$ be a demand, $\mathcal{U}$ an utility function as assessment criteria, and $\Omega_{\delta}^{+}$the set of all coverings given a set of available offers. The set of optimal coverings, denoted as $\Omega_{\delta, \mathcal{U}}^{+}$, is constituted of those covering sets which maximize the utility function $\mathcal{U}$.

$$
\Omega_{\delta, \mathcal{U}}^{+}=\left\{\Omega \in \Omega_{\delta}^{+} \mid \forall \Omega^{\prime} \in \Omega_{\delta}^{+} \cdot \mathcal{U}(\Omega) \geq \mathcal{U}\left(\Omega^{\prime}\right)\right\}
$$

Given the offers and demand in the previous example, the set of optimal coverings is $\left\{\left\{\omega_{1}, \omega_{5}\right\},\left\{\omega_{1}, \omega_{4}, \omega_{5}\right\}\right\}$.

Note that these covering sets have the same utility, although the latter seems to be redundant because values from $\omega_{5}$ override those from $\omega_{4}$. We can establish a preference order by means of any secondary assessment criteria, for example, by minimizing the number of offers. In this case, the optimal subset regarding $\min _{|\Omega|}$ of $\left\{\left\{\omega_{1}, \omega_{5}\right\},\left\{\omega_{1}, \omega_{4}, \omega_{5}\right\}\right\}$ is $\left\{\left\{\omega_{1}, \omega_{5}\right\}\right\}$.

\section{Related Work}

Figure 7 shows a brief comparison among related proposals, showing their characteristics on temporality at a first sight. Because of the limited extension of this paper, this section is devoted solely to temporal-aware proposals. A broader outline, which also includes the non-temporal-aware proposals, is available in [15, 18].

Note that our point of view is different from the perspective of service workflows, which is interested in the problem of finding an optimal execution plan of services in the context of a workflow [24]. We are interested in the procurement of web services whose demands and offers are temporal-aware. Of course, the workflow issue is very related to our problem, and they can be studied as a whole. 


\begin{tabular}{|c|c|c|c|c|c|c|}
\hline & 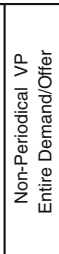 & 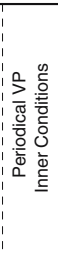 & 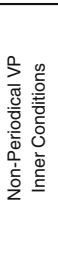 & 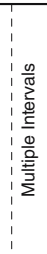 & 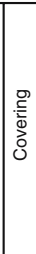 & 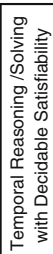 \\
\hline UDDle & $\mathrm{V}$ & & & & & \\
\hline WS-QoS & v & & & & & \\
\hline WSOL & & $\mathrm{v}$ & & & & \\
\hline WSLA & & & $\mathrm{v}$ & v & & \\
\hline WSML & v & V & & $v$ & & \\
\hline OWL-TIME & $\mathrm{v}$ & V & $\mathrm{v}$ & $v$ & & 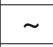 \\
\hline QRL & $\mathrm{v}$ & $\mathrm{V}$ & $\mathrm{V}$ & $\mathrm{V}$ & v & V \\
\hline
\end{tabular}

Fig. 7. A comparison among temporal-aware proposals

\subsection{Proposals Based on Ad-Hoc Formalisms}

These proposals do not have any formalism for temporal specifications, such as the UDDI Extension [20] and the WS-QoS ontology [22]. In general, they only allow to define an unique validity period for an entire demand or offer.

Fortunately, other proposals do allow to assign a validity period to every condition of a demand or an offer, such as the IBM WSLA Web Services Level Agreement language [6, 11] and the WSOL Web Service Offerings Language [23]. The HP WSML Web Services Level Agreement Management language [19] allows to specify both a single validity period for the entire agreement and also a periodic temporal interval to every condition. Both WSLA and WSML languages allow validity periods to be composed of multiple sub-intervals in distinct, limited ways as well.

\subsection{Proposals Based on Semantic Web}

These proposals are based on formalisms of the semantic web, having a much greater deal of expressiveness. The $O W L+T I M E$ Ontology [9, 10] is a very expressive language which is used by semantic-web-based approaches of WSP, such as the Web Ontology Language - Services $(O W L-S)[2,13,16]$.

However, having a greater deal of expressiveness leads to several computation problems of the Description Logics (DL) reasoners able to reason about such temporal specifications. As a matter of fact, in logics there exist a tradeoff between expressiveness and the computability of reasoning procedures [12], so the more expressive temporal DL languages are known to be undecidable, that is to say, there is no algorithm for computing the satisfiability of a DL specification. Most of temporal DL reasoners overcome this problem by making the language less expressive, or treating the time as a concrete domain in order to use hibrid reasoners so that temporal specifications are processed by external solvers, such as the CSP solvers. In general, both (1) the reasoning on less expressive temporal DL specifications, or (2) solving a CSP, are known to be NP-complete [1]. 


\section{Conclusions and Future Work}

In this paper, we have presented an approach to add temporal-awareness to WSP by using $\mathrm{CP}$, which endows our proposal with a declarative way to specify demands and offers so that the procurement tasks can be carried out by means of constraint satisfaction problems. We have introduced the notion of covering of a demand. We have also shown the need to review the semantics of procurement tasks if temporality is taken into account, and proposed a rigorous definition for them.

Our approach allows to specify a global validity period for a demand or an offer, and other validity periods which can be periodical or not, or composed of multiple intervals. These validity periods can be assigned to different conditions of the demand or the offer. Utility functions can be temporal-aware too, so that different utility values for a quality attribute can be defined at distinct time periods. The expressiveness of our approach is similar to semantic web-based proposals, though their major drawback is the undecidable nature of more complex temporal DL languages.

For future work, we are currently finishing the development of a proof-of-concept implementation, by adapting the prototype introduced in [18] so that it becomes temporal-aware. At operational level, consistency, conformance, and optimality have not to be computed at every time point of validity periods, just as they were defined in theory. A pre-processing step is needed in order to get the concrete time intervals of interest, then such tasks can be carried out on such time intervals.

Experiments need to be carried out in order to characterize the complexity of temporal-aware procurement tasks. As a result, it is expected to know what kind of temporal expressions to avoid because of their impact on the exponential behavior of CSP solving.

\section{References}

1. A. Artale and E. Franconi. A Survey of Temporal Extensions of Description Logics. Annals of Mathematics and Artificial Intelligence, 30(1-4):171-210, 2000.

2. The OWL Services Coalition. OWL-S: Semantic Markup for Web Services. Technical report, DARPA, 2004. http: / / www . daml . org.

3. A. Finkelstein and G. Spanoudakis. Software Package Requirements and Procurement. In Proc. of the $8^{\text {th }}$ Int'l IEEE Workshop on Software Specification and Design (IWSSD'96). IEEE Press, 1996.

4. X. Franch and J.P. Carvallo. Using Quality Models in Software Package Selection. IEEE Software, 20(1):34-41, 2003.

5. E.C. Freuder and M. Wallace. Science and substance: A challenge to software engineers. Constraints IEEE Intelligent Systems, 2000.

6. P. Grefen, H. Ludwig, and S. Angelov. A Three-Level Framework for Process and Data Management of Complex E-services. International Journal of Cooperative Information Systems, 12(1):455-485, December 2003.

7. P. Hentenryck. Constraint and Integer Programming in OPL. Informs Journal on Computing, 14(4):345-372, 2002.

8. P. Hentenryck and V. Saraswat. Strategic directions in constraint programming. ACM Computing Surveys, 28(4), December 1996. 
9. J. Hobbs and J. Pustejovsky. Annotating and Reasoning about Time and Events. In Proc. of the AAAI Spring Symposium on Logical Formalization of Commonsense Reasoning, Stanford, CA, March 2003.

10. J. Hobbs and J. Pustejovsky. An Ontology of Time for the Semantic Web. ACM Transactions on Asian Language Processing, Special Issue on Temporal Information Processing, 3(1):6685, March 2004.

11. Y. Hoffner, S. Field, P. Grefen, and H. Ludwig. Contract-driven Creation and Operation of Virtual Enterprises. Computer Networks, (37):111-136, 2001.

12. H.J. Levesque and R.J. Brachman. Expressiveness and Tractability in Knowledge Representation and Reasoning. Computational Intelligence, 3(2):78-93, May 1987.

13. L. Li and I. Horrocks. A Software Framework for Mathmaking based on Semantic Web Technology. In Proc. of the $12^{\text {th }}$ ACM Intl. Conference on World Wide Web (WWW'03), pages 331-339, 2003.

14. K. Marriottt and P.J. Stuckey. Programming with Constraints: An Introduction. The MIT Press, 1998.

15. O. Martín-Díaz, A. Ruiz-Cortés, A. Durán, D. Benavides, and M. Toro. Automating the Procurement of Web Services. In $1^{\text {st }}$ Int.l Conf. on Service-Oriented Computing, volume 2910 of LNCS, pages 91-103, Trento, Italy, 2003. Springer Verlag.

16. F. Pang and J. Hobbs. Time in OWL-S. In Proc. of the AAAI Spring Symposium on Semantic Web Services, pages 29-36, Stanford, CA, 2004.

17. A. Ruiz-Cortés. A Semiqualitative Approach for the Automatic Management of Quality Requirements (in Spanish). PhD thesis, University of Seville, 2002.

18. A. Ruiz-Cortés, O. Martín-Díaz, A. Durán, and M. Toro. Improving the Automatic Procurement of Web Services using Constraint Programming. Int. Journal on Cooperative Information Systems, 14(4):439-467, December 2005.

19. A. Sahai, V. Machiraju, M. Sayal, L.J. Jin, and F. Casati. Automated SLA Monitoring for Web Services. Research Report HPL-2002-191, HP Laboratories, 2002.

20. A. ShaikhAli, O. Rana, R. Al-Ali, and D. Walker. UDDIe: An Extended Registry for Web Services. In Proc. of the IEEE Int'l Workshop on Service Oriented Computing: Models, Architectures and Applications at SAINT Conference. IEEE Press, January 2003.

21. K. Sycara, M. Klusch, S. Widoff, and J. Lu. Dynamic Service Matchmaking among Agents in Open Information Environments. SIGMOD Record, 28(1):47-53, 1999.

22. M. Tian, A. Gramm, T. Naumowicz, H. Ritter, and J. Schiller. A Concept for QoS Integration in Web Services. In Proc. of the IEEE Int'l Web Services Quality Workshop (at WISE'03), pages 149-155, 2003.

23. V. Tosic, K. Patel, and B. Pagurek. Reusability Constructs in the Web Service Offering Language (WSOL). Research Report SCE-03-21, The Department of System and Computer Engineering, Carleton University, Ottawa, Canada, 2003.

24. L. Zeng, B. Benatallah, A.H.H. Ngu, M. Dumas, J. Kalagnanam, and H. Chang. QoS-Aware Middleware for Web Services Composition. IEEE Transactions on Software Engineering, 30(5):311-327, May 2004. 